\title{
Development of Automated Camera Platform for Optimization of Machine Vision with IoT
}

\author{
Alvin Yap Chee Wei, Alex Haw Wai Kit, Gabriel Yosua Rantung
}

\begin{abstract}
Most industrial cameras lack the ability to perform auto-focus function and this pose a problem to picture analytics. As a result, pictures taken are unclear, and this pose a problem for analytics. This paper describes the process of developing an automated camera platform based on two axis movement. The design of the platform has been produced with a $3 D$ model, and the model was further developed into working system. The system is controlled by using a Delta programmable logic controller (PLC) and programmed with WPLSoft using ladder logic. Two linear actuators were used to move the camera from one position to another position and the lighting system was also designed to improve the lighting environment for the camera. The system was tested for accuracy and repeatability on the position and the speed of the linear actuator. The result shows that the system is applicable and reliable. IoT has been an added advantage to the system for better control. The implementation of automated camera platform is therefore realistic with over $95 \%$ accuracy in terms of position and rotation.
\end{abstract}

Keywords-automated camera platform, programmable logic controller (PLC), linear actuator, Internet of Things (IoT).

\section{INTRODUCTION}

Automated system plays an important role in a variety of fields such as manufacturing, construction, automobile and basic human daily life. Automation has been gaining popularity as machines have better movement speed, higher accuracy, higher efficiency, and higher consistency and produces the part of higher quality compared to human.

The purpose of this project is to create an automated camera platform for machine vision where machine vision is used to detect the glove defect while the automated camera platform will move the camera upward, downward, forward and backward. The control of the machine for camera movements is also controllable using smartphone with the aid of IoT. Therefore, automated camera platform will handle the camera while machine vision will detect the faulty glove.

\section{LITERATURE REVIEW}

For the control system, PLC was being used as a control system as it can be used for motion control, but there are different methods that were used to control the motion or speed. One of the researches from the literature review used open loop system to move the solar panel to specific angle by altering the sun-tracking frequency using pre-programmed

Revised Version Manuscript Received on 10 September, 2019.

Alvin Yap Chee Wei, School of Engineering, Asia Pacific University, Kuala Lumpur, Malaysia. (Email: yap.alvin@staffemail.apu.edu.my)

Alex Haw Wai Kit, School of Engineering, Asia Pacific University, Kuala Lumpur, Malaysia.

Gabriel Yosua Rantung, School of Engineering, Asia Pacific University, Kuala Lumpur, Malaysia. according to the azimuth solar velocity theory in different time and date [1]. The control of the solar panel using PLC was able to increase the solar energy. Another research from the literature review shows that the efficiency of induction motors was improved by using PLC for intelligent control on a three-phase induction motor and DC generator [2]. By combining these methods, the speed of induction motor able to be varied by using PLC. Besides that, another research showed that using library from Omron programming software makes the motion control programming simpler, however, it is applicable only for machines that are simple, and the motion control accuracy tends to be low [3].

For mechanism, some research shows that stepper motor was used to control the rotating angle or control the motion [2]. On the other hand, one of the researchers used linear actuator and damper system to control the tilt axis in rotation [4]. By using this actuator and technique, the research shows that the rotational type of tripod parallel mechanism has larger tilt angle, high position accuracy and better stability compared to Stewart-Gough platform. Another research shows that the use of stepper motor to control the ball screw is being considered as the most suitable as ball screw requires less torque to move in and out while the rotary motion of a stepper motor can be converted to linear motion for various application where less torques is being needed [5].

Research has been done on illumination for image processing. Illumination system is important as it produces significant difference between the object, reflectance estimation and the background [1]. The result from the study shows that by adding the light source, the moving object which is having a speed of $106 \mathrm{rpm}$ can be detected by the camera and it also have $94.264 \%$ accuracy in image acquisition. Besides that, another research shows that using SRAM to collect the scattered light and bright field, the image processing was able to detect the defects on an object such as a missing pattern on a circuit board [6]. Another study shows that multiple camera-based NIR-illuminator is needed to detect the object under different light intensity [7].

For anti-vibration system, literature review had been done on few researches. One research shows that a rubber suspension can be used to reduce the magnitude of the vibration, and during the research, the researcher found out that the rubber suspension can also be used to reduce the noise level [8]. Besides that, another research shows that vibration absorber is not only to reduce the vibration. By adding a piezoelectric to the vibration absorber, it is possible to harvest energy from the vibration [9]. Moreover, 


\section{DEVELOPMENT OF AUTOMATED CAMERA PLATFORM FOR OPTIMIZATION OF MACHINE VISION WITH IOT}

anti-vibration can also help to improve the air flow in a system by using time delay in repetitive control [10].

Smartphone is a good device for digital control on the machine as well as the IoT implementation, and user's interaction as it has the capability to get connected easily using Wi-Fi, Bluetooth, cellular data, etc. [11]. A study shows that Android smartphone can control a house system using IoT that is integrated with $\mathrm{Wi}-\mathrm{Fi}$ as its communication protocol and even can reduce the cost of operation [12].

\section{DESIGN METHODOLOGY}

In this section, the 3D model will be shown and explained. Further, an explanation on the working principle will be provided.

\section{A. 3D Model Design}

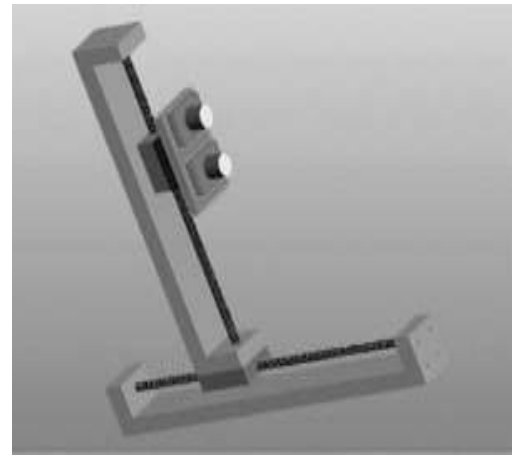

Fig. 1. 3D model design for automated camera platform

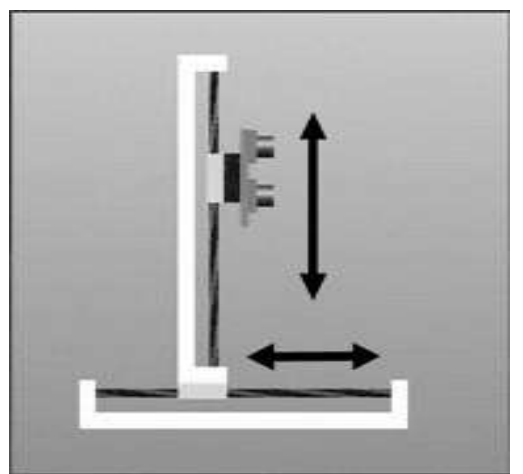

Fig. 2. Movement of both linear actuators

Fig. 1 shows the 3D design of the automated camera platform and the components used in building the system of are linear actuator with servo motor and lighting system.

Two linear actuators were used to move the camera upward, downward, forward and backward as shown in Fig. 2. For the lighting system, a led that is capable to display red, green, and blue (RGB) was used to improve the lighting environment for the camera system to detect the defective glove.

\section{B. Design Principle}

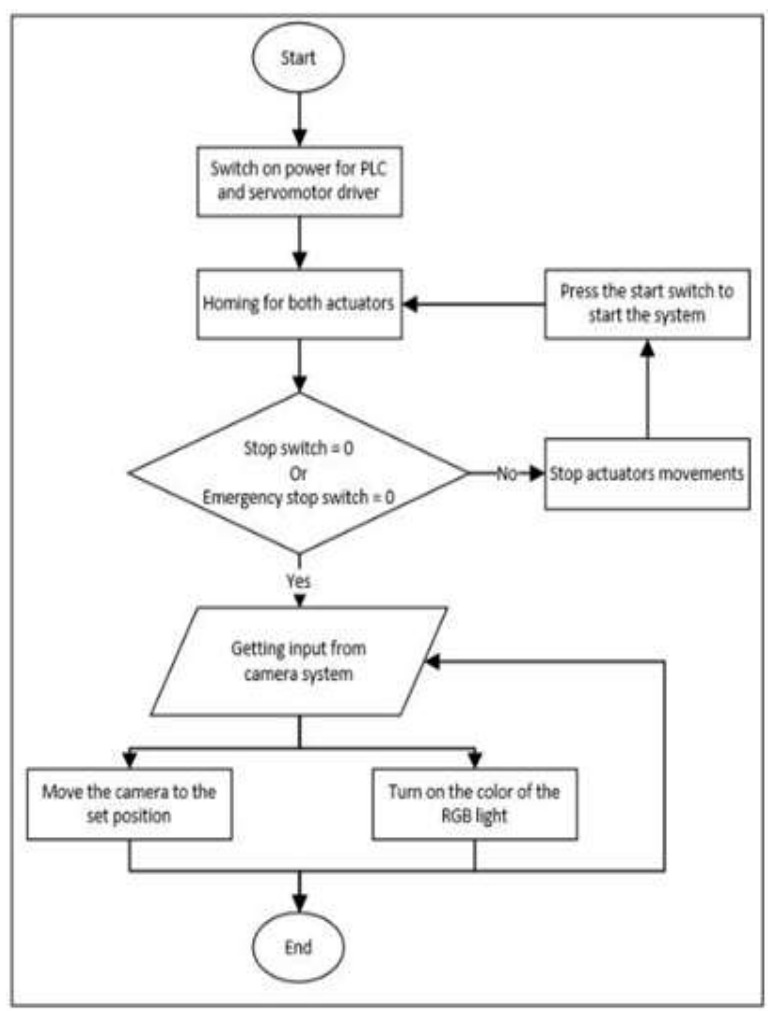

Fig. 3. Flowchart for automated camera platform

Fig. 3 shows the flowchart of Automated Camera Platform, whereby the system begins when the all the MSB in the electrical panel is switch on. Once the MSBs switched on, both actuators will do 'homing' process. 'Homing' process is where the actuator will be moved back to the original position so that all the current reading will be reset back to zero and it is usually known as initialization.

Once homing process is done, the PLC will check on the reading of the Emergency Stop switch and Stop switch. If both switches are off, the PLC will collect information from the camera system which are the size and color of the glove. Then both actuators will move the camera position to the pre-set position of the camera. At the same time, the color of the RGB led will changed accordingly.

If the Stop switch or the Emergency Stop switch is pressed, both actuators will stop moving. To resume the operation of the system, 'Start' switch must be pressed. After pressing the Start switch, both actuators will do homing process to reset the current reading. Once it has been turned into reset, the PLC will continue the process of collecting data for size and color of the glove from the camera system.

The setting of the servo drive is being done using a software known as ASDA Soft. This software is used to set the function of all the digital inputs and outputs of the servo drive. For example, in this project, one of the digital inputs was set to that when 'On' signal is received, it will move the actuator back to the homing position. 


\section{PROGRAM DESIGN}

The ladder logic program of PLC is being discussed as follows.

\section{A. Setup Communication Between PLC and Servo Drive}

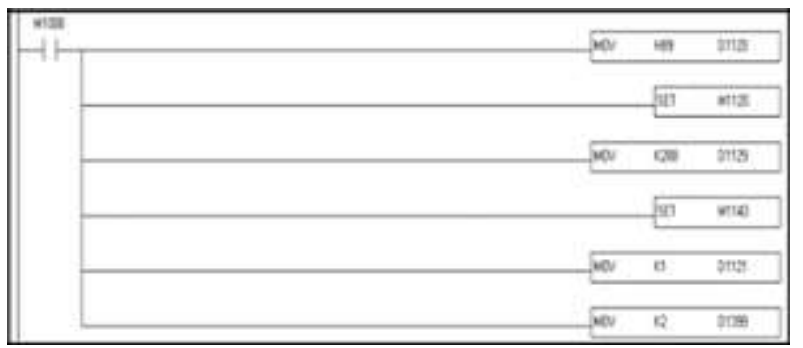

Fig. 4. Setting for communication between PLC and servo drive always 'On' switch. All the outputs in Fig. 4 are to setup the communication between the PLC and servo drive by setting the servo drive 1 and 2 as SlaveID\#1 and SlaveID\#2. Besides that, it is also used for the communication protocol to retain it with the servo drive through RS-485.

\section{B. ID Setting 1}

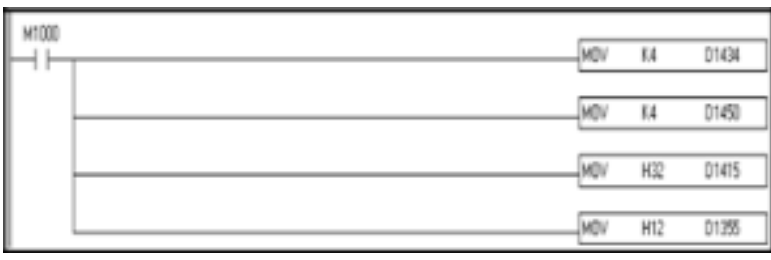

Fig. 5. ID Setting for SlaveID\#1

Fig. 5 shows the ID setting for the servo drive 1. This setting is to set the data length to be read and written on SlaveID\#1. Based on Fig. 5, the data length to be read and written is 4 . 'D1415' is the starting reference address for the Master which is the PLC to write in SlaveID\#1. For the 'D1355' is the starting reference address for the Master to read data from SlaveID\#1.

For the setting for SlaveID\#2, the same approach was being used with a different address that was set. The difference of setting for data length to be read and written on SlaveID\#2 is that for 'D1434' was changed to 'D1435' and 'D1450' was changed to 'D1451'. Meanwhile the starting reference for SlaveID\#2 was changed from 'D1415' to 'D1416' and from 'D1355' to 'D1356'.

\section{Setup Position}

\begin{tabular}{|c|c|c|c|}
\hline vim & $\sqrt{\operatorname{sen} y}$ & $2+4$ & $\overline{p r m}$ \\
\hline -1/2- & & & 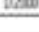 \\
\hline-15 & DMON & Dtax & $\mathrm{Dax} 2$ \\
\hline wites & & & \\
\hline$-1 \pi-$ & $\sqrt{\sec 20}$ & 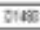 & 020 \\
\hline w' & & & \\
\hline - it & $\sqrt{\sin 3}$ & otas & D.206 \\
\hline xega & $\sqrt{\mathrm{Fu} x}$ & गुद & Dons \\
\hline wits. & & & \\
\hline - it & PMDS & $574 x$ & 82010 \\
\hline mot & & & \\
\hline 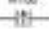 & - Tusy & $D 168$ & $D 272$ \\
\hline
\end{tabular}

Fig. 6. Setting for position

Fig. 6 shows on the method on how to save the position of the camera using ladder logic with assembly language in WPLSoft for SlaveID\#1. The input for this setting is the
Fig. 4 shows the 'M1000' in WPLSoft software acts as

rising edge. The function of rising edge is that it will only on once when pressed. The method to on the rising edge is using PC. 'DMOV' function is to move the double word function. The method on using this function is that after 'DMOV', the ' $\mathrm{D} 1480$ ' is the data source and the 'D2000' is the data destination. The same method was used for SlaveID\#2 but with different data destination address and different data source address.

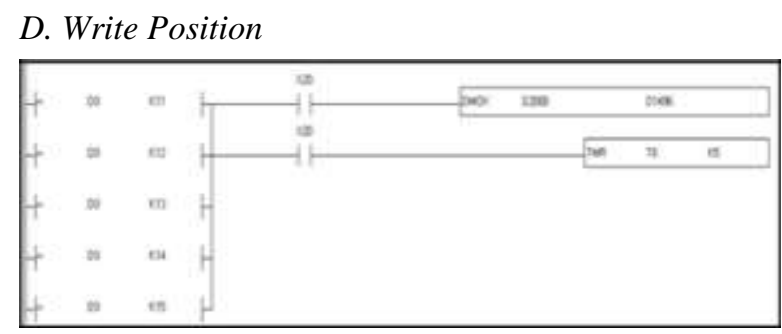

Fig. 7. Write data position to servo drive

Fig. 7 shows how the data received from the camera system was being compared and being sent to SlaveID\#1. The input is using the comparison function where the data received from camera, 'D0' was compared with the set value. When the value matches the set value, it will send the data saved in 'D2000' into the servo drive to move the camera. The data send is in the form of pulse since servo motors move according to the pulse. For this servo drive and motor, one pulse is equivalent to $0.01 \mathrm{~mm}$. Therefore, every single pulse that is sent into the servo drive will command the servo motor to move $0.001 \mathrm{~mm}$. The first digit of the number represents the position. Therefore, when the first digit is 1 , the actuator will move the camera to position 1 based on the saved data. For the timer function, TMR has a function to delay the movement of the actuator by 5 seconds to prevent overlapping data. X20 is one of the jog switches where it is used to determine whether the system will work automatically or not. Based on Fig. 7, the system will only work under the condition where setting will be sent into the PLC if the switch is on. Otherwise, the system will manually use the jog switch to move the actuator. The same setting will be used for the other position with different data source address.

For SlaveID\#2, the input and output setting will look the same as SlaveID\#1. The only difference between SlaveID\#2 and SlaveID\#1 is the address of data source and data destination. On SlaveID\#1, the address of data source starts from D2000 to D2012, while SlaveID\#2 starts from D2014 to D2026. The address of data destination will be changed from D1496 to D1528.

\section{E. Setting for Speed and Position Data Collection}

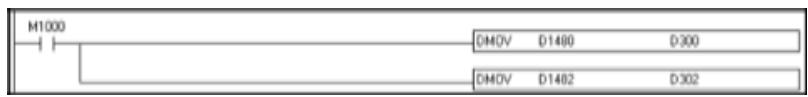

Fig. 8. Setting to collect speed and position data

Fig. 8 is the method that is used to read data from SlaveID\#1. The D300 is used to read the pulse. Then, the pulse data can be saved using the rising edge method to save 


\section{DEVELOPMENT OF AUTOMATED CAMERA PLATFORM FOR OPTIMIZATION OF MACHINE VISION WITH IOT}

the position of the camera, while the D302 is to read the speed of the servo motor in revolution per minute (rpm). The only disadvantage is that the reading of rpm is difficult to be obtained as the travel distance of the actuator is very short. Therefore, the required time for the actuator to move to the specified position will be short. While for SlaveID\#2, the ladder logic will be the same as SlaveID\#1, however, the difference is that the data destination and data source address will be different from SlaveID\#1.

\section{F. Setting for Speed of Actuator}

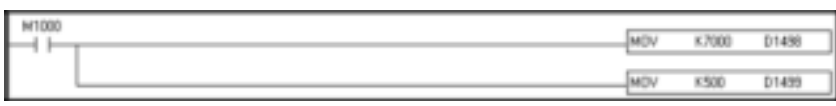

Fig. 9. Setting for speed data

The setting of speed data for the servo motor of SlaveID\#1 is programmed using ladder logic as shown on Fig. 9. The speed in rpm of the servo motor is being set as $700 \mathrm{rpm}$. The data of K7000 is the data for the servo drive as 1 data is equals to $0.1 \mathrm{rpm}$. The D1498 is to set the rpm for the automatic function where camera will be moved according to the data received from the camera system. The D1499 is to set the speed for manual where the actuator is being controlled using jog switch. The purpose of using $50 \mathrm{rpm}$ for jog is because of the use of jog is to obtain the most suitable position for the camera according to the size of the detected glove. The difference between SlaveID\#2 and SlaveID\#1 here is the address of data destination.

G. Jog Switch Setting

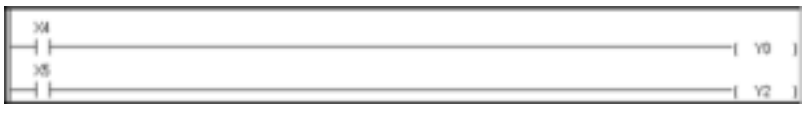

Fig. 10. Setting for jog switch

Fig. 10 shows the setting of jog switch for SlaveID\#1. The ladder logic program will be straight forward as it does not require any data transfer since the only data that is needed to be transferred is the speed data. By pressing the $\mathrm{X} 4 \mathrm{jog}$ switch, which is labelled as forward jog switch, the actuator will move forward as $\mathrm{Y} 0$ is being connected to the digital input of servo drive 1 . When an 'on' signal is sent to $\mathrm{Y} 0$, it will move the camera with the speed of $50 \mathrm{rpm}$, while the X5 and $\mathrm{Y} 2$ is for the reverse jog function. At the same time, $\mathrm{Y} 2$ is being connected to another digital input of the servo drive 1 . All the settings of the servo drive were being set using ASDA Soft software. The similar method was used for servo drive 2 with different switches and different output to servo drive 2 digital input.

\section{H. Setting for Start, Stop and Emergency Stop Switch}

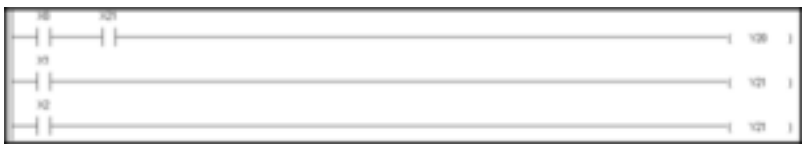

Fig. 11. Settings for start, stop and emergency stop switches

Fig. 11 shows how the start, stop and emergency stop switches that are being programmed in the PLC ladder logic. Based on the wiring diagram, the $\mathrm{X} 0$ is the start switch, $\mathrm{X} 21$ is the manual switch and Y20 is the digital input of both servo drives. The function of $\mathrm{X} 0$ will work if the $\mathrm{X} 21$ is turned on.
When $\mathrm{X} 0$ is pressed, the $\mathrm{Y} 20$ which has been set as the homing function in ASDA Soft will move both actuators back to their original position.

$\mathrm{X} 1$ and $\mathrm{X} 2$ are the stop and emergency stop switches. When either one of these switches is being pressed, the Y21 will be turned on. Y21 is the digital input of both servos drives where in ASDA Soft setting, if Y21 is turned on, it will stop the movement of the actuators.

\section{SYSTEM IMPLEMENTATION}

\section{A. Overall Hardware}

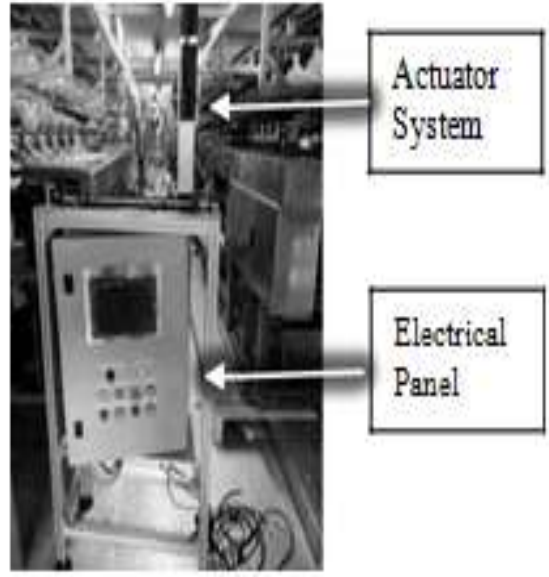

Fig. 12. Overall picture of system

Fig. 12 is the overall hardware built. There are two actuators on top of the profile table, and in the middle of the profile is the electrical panel. On the surface of the panel, there are touch panel and several switches.

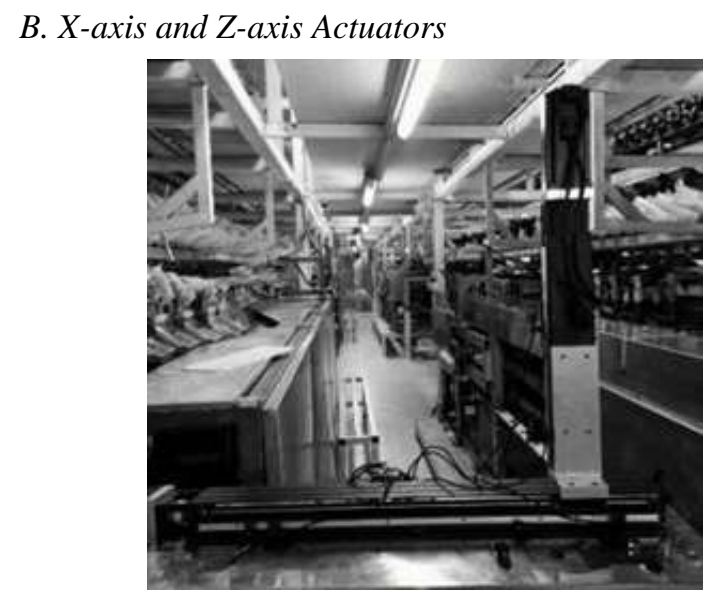

Fig. 13. Actuator system

As shown on Fig. 13, the actuators were being installed in the way that the camera can be moved in four directions which are upward, downward, forward and backward. The $\mathrm{Z}$-axis actuator has the shortest working length that when the $\mathrm{X}$-axis actuator moves, it will not cause high vibration to the $\mathrm{Z}$-axis actuator. 


\section{Front Panel}

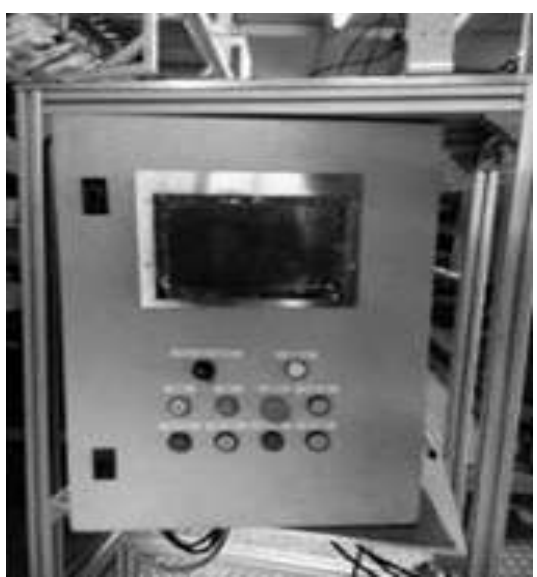

Fig. 14 Electrical panel touch panels. The switches which are mounted are the start, stop, emergency stop and jog switches.

D. Wiring in Electrical Panel

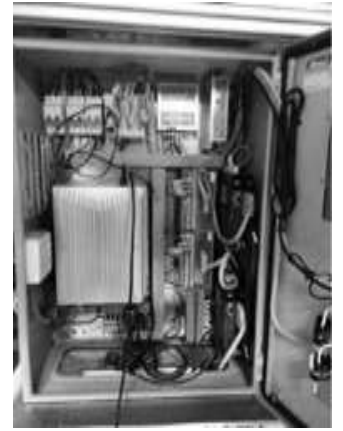

Fig. 15 Components in electrical panel

Fig. 15 is the electrical panel where all the components are mounted such as the miniature circuit breakers (MCBs), power supply unit (PSU) of $12 \mathrm{~V}$ and $24 \mathrm{~V}$, PLC with expansion card, servo drives, ventilation fan, socket and industrial PC.

\section{E. IoT Implementation on the Machine}

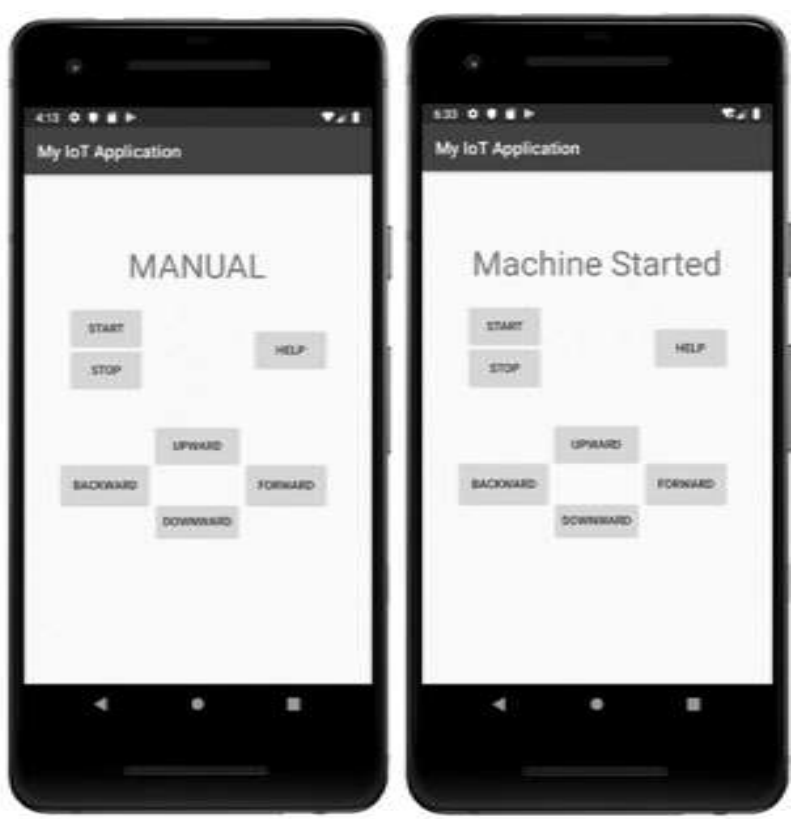

Fig. 16. IoT implementation
Fig. 14 above is the panel where there are few switches and

The machine is controllable using Android smartphone that is linked with the IoT implementation. The smartphone is installed with the control application as shown in Fig. 16 is connected to the internet via Wi-Fi to control the machine. As well as the machine, it is connected to the industry's open platform communication (OPC). Using Wi-Fi internet connection, the smartphone device is linked to the OPC that makes it capable to control the machine.

The Android application is capable to control the mechanical movements of the machine using user interface that is defined by the user whether the machine should go upward, downward, forward and backward.

\section{RESULTS AND ANALYSIS}

A series of tests were conducted on the hardware system in order to determine its performance and to verify the usage reliability.

A. Test on Accuracy and Repeatability Performance for Position 1

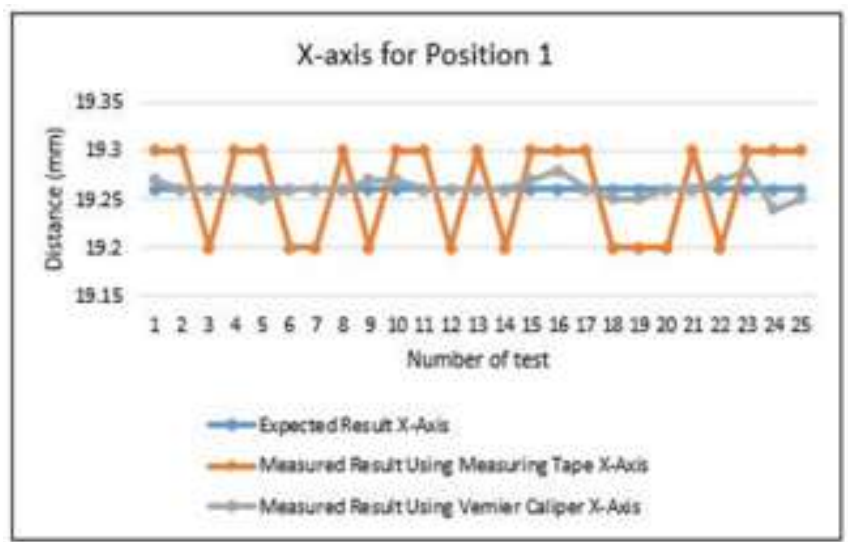

Fig. 17 Result of $\mathrm{X}$-axis for position 1

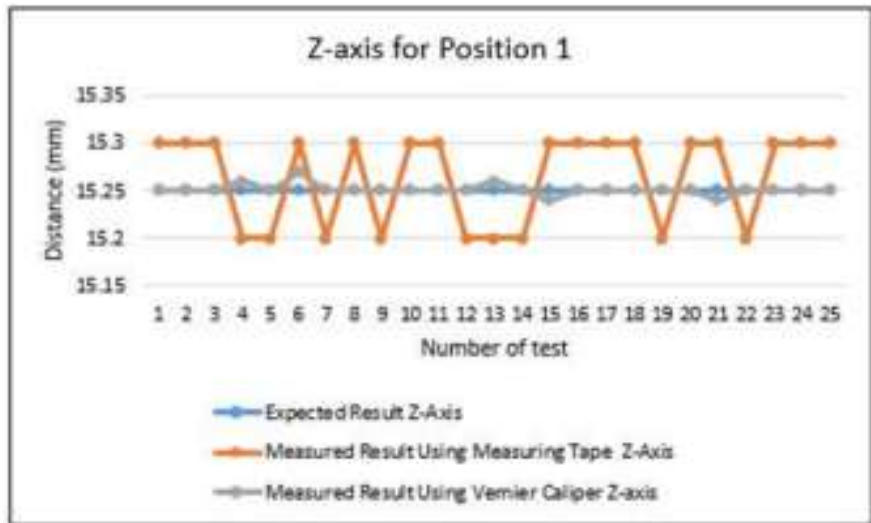

Fig. 18 Result of Z-axis for position 1

Based on Fig. 18 and Fig. 19, the use of Vernier caliper to measure the distance moved by the actuator from the original position is because of the measuring tape scale is smaller compared to Vernier caliper as the scale movement of the linear actuator is as small as $0.01 \mathrm{~mm}$ per pulse. According to the measured results from Vernier caliper, the accuracy and the repeatability of the actuator to move the camera back to

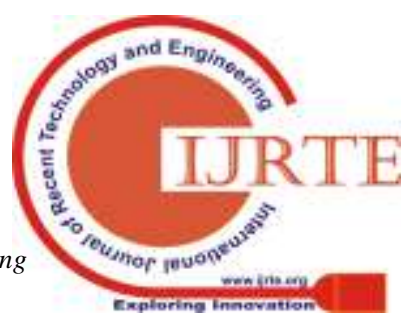




\section{DEVELOPMENT OF AUTOMATED CAMERA PLATFORM FOR OPTIMIZATION OF MACHINE VISION WITH IOT}

the specified position is high, with only few differences that are between $0.01 \mathrm{~mm}$ or $0.02 \mathrm{~mm}$.

According to the graph, the results obtained from the measuring tape of $\mathrm{X}$-axis and $\mathrm{Z}$-axis for position 1 ranged from $19.2 \mathrm{~mm}$ to $19.3 \mathrm{~mm}$ and $15.2 \mathrm{~mm}$ to $15.3 \mathrm{~mm}$. As comparison, the results obtained using Vernier caliper for $\mathrm{X}$-axis and Z-axis are from the range of $19.25 \mathrm{~mm}$ to 19.27 $\mathrm{mm}$ and $15.24 \mathrm{~mm}$ to $15.27 \mathrm{~mm}$.

\section{B. Test on Rotational Speed Performance of Actuators}

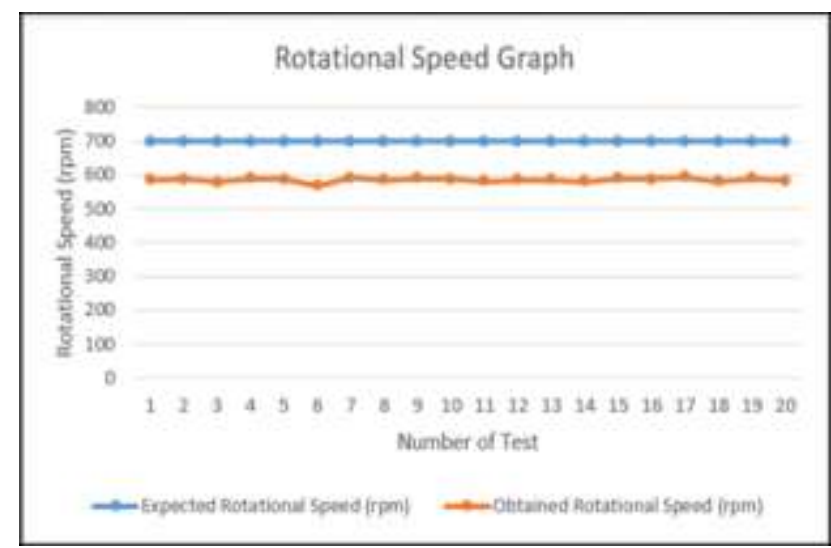

Fig. 19. Result of Rotational Speed

Fig. 19 has the graphs of the results obtained from PLC using the speed data collection method. According to the graphs, the results obtained from the PLC and the preset rotational speed of the actuator are having a huge difference. The reason on the difference is because the Delta servo drive is having a feature where it has used to smoothen the movement of the actuator. The purpose of smoothing is to prevent the sudden start and stop of the maximum rotational speed as of the sudden start and stop will cause a huge vibration and internal damage to the linear actuator, servo motor and the camera itself.

\section{CONCLUSION}

This project has demonstrated the usability of the automated camera platform. The automated camera platform can be moved to the specified location in high accuracy and repeatability with the proven method of using Vernier caliper. Besides, the rotational speed of the linear actuator with servo motor was examined and the reason on the differences had been explained. Furthermore, the IoT implementation using smartphone to control the machine has been explained. Therefore, the overall results of this system had showed the reliability and sustainability of this system.

\section{ACKNOWLEDGEMENT}

The authors would like to thank Asia Pacific University of Technology and Innovation for partial funding in developing the system through Research and Development Grant.

\section{REFERENCES}

1. Zhe, M. et al., (2016). Open-loop solar tracking strategy for high concentrating photovoltaic systems using variable tracking frequency. Energy Conversion and Management, 117(1 June 2016), pp. 142-149.

2. Yilmazlar, E., Erdemir, V., Kuscu, H. \& Gullu, A., (2018). Design Of Stepper Motor Control Interface With
Embedded Systems. International Journal Of Engineering Research And Development , 14(6), pp. 17-22.

3. Omron Packaging Department (2016), Simple motion control implementation with Omron PLC. Europe, Omron.

4. Cho, S. W. et al., (2018). Face Detection in Nighttime Images Using Visible-Light Camera Sensors with Two-Step Faster Region-Based Convolutional Neural Network. Molecular Diversity Preservation International and Multidisciplinary Digital Publishing Institute (MDPI), 18(9), p. 2995.

5. Cloutier, J., (2014). Simulation and Control of a Ball Screw System Actuated by a Stepper Motor with Feedback, Guelph, Ontario: The University of Guelph.

6. Cahill, P. et al., (2018). Data of piezoelectric vibration energy harvesting of a bridge undergoing vibration testing and train passage. Data in Brief, Volume 17, pp. 261-266.

7. Luo, R. K., (2017). Impact simulation and experiment on rubber anti-vibration systems. Polymer Testing, Volume 50, pp. 335-342.

8. Tu, G. J., Karstoft, H., Pedersen, L. J. \& Jorgensen, E., (2015). Illumination and Reflectance Estimation with its Application in Foreground Detection. Molecular Diversity Preservation International and Multidisciplinary Digital Publishing Institute (MDPI), Volume 15, pp. 21407-21426.

9. Coetzer, B. \& Gouws, R., (2016). Design of a PLC-based variable load, speed control system for a three-phase induction motor. Vereeniging, South African Universities Power Engineering Conference (SAUPEC).

10. Noguchi, Y., Nakamura, Y. and Wakui, S. (2014) Repetitive control for pneumatic anti-vibration apparatuses under a multi-loop control system. 2015 IEEE International Conference on Industrial Technology (ICIT).

11. El Khaddar, M. A. \& Boulmalf, M., 2017. Smartphone: The Ultimate IoT and IoE Device. In: Smartphones from an Applied Research Perspective. Rijeka: InTech, pp. 137-162. 